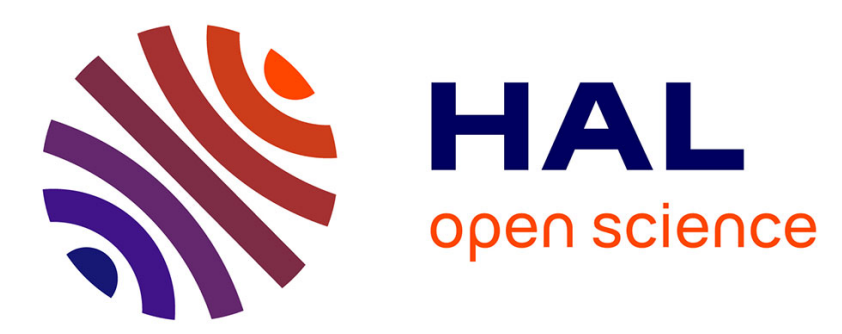

\title{
Modeling circadian clock-cell cycle interaction effects on cell population growth rates
}

\author{
Raouf El Cheikh, Samuel Bernard, Nader El Khatib
}

\section{To cite this version:}

Raouf El Cheikh, Samuel Bernard, Nader El Khatib. Modeling circadian clock-cell cycle interaction effects on cell population growth rates. Journal of Theoretical Biology, 2014, 363, pp.318-331. 10.1016/j.jtbi.2014.08.008 . hal-01055081

\section{HAL Id: hal-01055081 \\ https://hal.science/hal-01055081}

Submitted on 11 Aug 2014

HAL is a multi-disciplinary open access archive for the deposit and dissemination of scientific research documents, whether they are published or not. The documents may come from teaching and research institutions in France or abroad, or from public or private research centers.
L'archive ouverte pluridisciplinaire HAL, est destinée au dépôt et à la diffusion de documents scientifiques de niveau recherche, publiés ou non, émanant des établissements d'enseignement et de recherche français ou étrangers, des laboratoires publics ou privés. 


\title{
Modeling circadian clock-cell cycle interaction effects on cell population growth rates
}

\author{
R. El Cheikh ${ }^{\mathrm{a}, \mathrm{b}}$, S. Bernard ${ }^{\mathrm{a}, \mathrm{b}}$, N. El Khatib ${ }^{\mathrm{c}}$ \\ ${ }^{a}$ CNRS UMR 5208, Institut Camille Jordan, Université Lyon 1, 43 blvd. du 11 \\ novembre 1918, F-69622 Villeurbanne cedex, France \\ ${ }^{b}$ DRACULA Inria Grenoble Rhône-Alpes, Montbonnot F-38322, France \\ ${ }^{c}$ Lebanese American University, Department of Computer Science and Mathematics, \\ Byblos, P.O.Box 36, Byblos, Lebanon
}

\section{Abstract}

The circadian clock and the cell cycle are two tightly coupled oscillators. Recent analytical studies have shown counter-intuitive effects of circadian gating of the cell cycle on growth rates of proliferating cells which cannot be explained by a molecular model or a population model alone. In this work, we present a combined molecular-population model that studies how coupling the circadian clock to the cell cycle, through the protein WEE1, affects a proliferating cell population. We show that the cell cycle can entrain to the circadian clock with different rational period ratios and characterize multiple domains of entrainment. We show that coupling increases the growth rate for autonomous periods of the cell cycle around $24 \mathrm{~h}$ and above $48 \mathrm{~h}$. We study the effect of mutation of circadian genes on the growth rate of cells and show that disruption of the circadian clock can lead to abnormal proliferation. Particularly, we show that Cry1, Cry2 mutations decrease the growth rate of cells, Per2 mutation enhances it and Bmal1 knockout increases it for

Email address: nader.elkhatib@lau.edu.lb (N. El Khatib) 
autonomous periods of the cell cycle less than $21 \mathrm{~h}$ and decreases it elsewhere. Combining a molecular model to a population model offers new insight on the influence of the circadian clock on the growth of a cell population. This can help chronotherapy which takes benefits of physiological rhythms to improve anti-cancer efficacy and tolerance to drugs by administering treatments at a specific time of the day.

Keywords: circadian clock, cell cycle, chronotherapy, age-structured equations

\section{Introduction}

The circadian clock regulates body daily rhythmic activities, from the wake-sleep phases succession and hormone production to blood pressure and body temperature. Several epidemiological studies have shed light on the fact that individuals with disrupted circadian rhythms have increased risk of developing tumorigenic diseases $[1,2,3,4,5,6,7]$. Studies made on yeast revealed restriction of cell division to the reductive phases of the yeast metabolic cycle. This type of control was shown to be involved in circadian regulation and may be a general strategy for the robust maintenance of cellular processes. This regulation insures that the cell cycle evades the potentially mutagenic redox environment of the oxidative respiratory phase, helping to minimize the occurrence of futile reactions $[8,9]$.

The circadian clock interacts with the cell cycle through multiple molecular pathways $[10,11,12,13,14,15]$. Hence, a disruption of the circadian clock can lead to abnormal cell proliferation and enhances tumor development.

Circadian rhythms are generated at the cellular level by a finely regu- 
lated gene network that produces sustained $24 \mathrm{~h}$ period oscillations in clock gene and protein expression. This network involves several genes and relies on transcriptional, translational and post-translational mechanisms. Oscillations arise from an autoregulatory negative feedback loop system in which a clock protein inhibits the expression of its own gene by inactivating a transcription factor $[16,17,18,19,20,21,22]$.

The cell cycle is usually divided into four phases G1, S, G2, M. Progression through each phase depends on the activity of cyclins and cyclin-dependent protein kinase complexes $(\mathrm{Cdks})$ and a mitosis promoting factor (MPF). When MPF activity is high, the cell progresses through the cycle. When it is low, progression stops [23]. Each phase of the cell cycle is controlled by a different cyclin/Cdk complex: G1 is controlled by cyclin D/Cdk4-6, G1/S transition by cyclin E/Cdk2, S phase by cyclin A/Cdk2 and G2/M transition by cyclin B/Cdk1 [14].

The circadian clock and the cell cycle are tightly connected. The circadian clock gates the cell cycle through the regulation of different Cdks. It has been reported that BMAL1/CLOCK activates the transcription of the kinase WEE1 to regulate the G2/M transition [24]. The circadian clock, via the protein $\mathrm{REV}-\mathrm{ERB} \alpha$, regulates the transcription of $\mathrm{p} 21$, which inhibits Cdk2 and blocks the G1/S transition [7]. The circadian clock is also involved in direct control of DNA damage and apoptosis pathways by virtue of its regulation of Chk2 and other related factors [6, 25].

Two main approaches have been used to model the coupling between the cell cycle and the circadian clock oscillators. The first approach is to model the molecular machinery of the cell. It is usually based on ordinary 
differential equations, where the variables describe the intracellular molecular concentrations of both oscillators. Chauhan and colleagues constructed such a model to account for the regulation of mammalian cell cycle progression and its gating by the circadian clock in the regenerating liver [26]. Zamborszky and colleagues used a minimal model for circadian rhythms coupled to a cell cycle model that had been originally developed for the yeast cell cycle. Their model revealed quantized cell cycles and they suggested that cell size control is influenced by the clock [27]. More recently, Gérard and colleagues used a detailed computational model for the Cdk network driving the mammalian cell cycle to study the effect of multiple molecular links to the circadian clock [28]. They characterized the domains of autonomous periods where the cell cycle can be brought to oscillate to 24 or $48 \mathrm{~h}$ periods, and determined conditions for switching between these two patterns of entrainment.

The second approach is to model a cell population, leaving aside molecular details. This approach is based on PDEs, especially the category of physiologically-structured models, or on individual-based models and cellular automata. In these models, the cell cycle is divided into multiple, discrete phases and the circadian clock is coupled via time-periodic parameters, such as the transition coefficients or phases duration. Altinok and colleagues used a cellular automaton model to examine the entrainment of the cell cycle by the circadian clock [29]. Clairambault and colleagues used an age-structured PDE system to model a population of cells under the control of the circadian clock $[30,31]$. The circadian clock was taken into account through periodic cell cycle phase transition coefficients into the equations.

Compared to population models, molecular models capture more details 
of the fine regulation of the cell cycle, and in particular, can predict the effect of mutations on the cell cycle regulation. However, molecular models rarely describe explicitly dividing cell populations and it is not clear how growth rates are affected by disruptions at the molecular level.

Here, we present a mathematical model that combines the molecular and the population levels, to study the influence of the circadian clock on the growth of a population of cells. We study the influence of circadian clock gene mutations on the net growth rate of a dividing population. We show that disruption of circadian rhythms can lead to abnormal proliferation. Depending on autonomous cell cycle properties and the nature of the disruption, circadian clock gene mutations can lead to faster or slower growth rates. We characterize the effect of circadian clock gene mutations, and show that combined molecular/population model brings to the dynamics of cell proliferation a picture more complete than a molecular model alone.

\section{Coupling the cell cycle and the circadian clock}

Becker-Weimann and colleagues developed a simple model that takes into consideration molecular information and analyzed the roles of feedback loops on the oscillatory dynamics [32]. This model was used to explore the role of the negative feedback loop created by the transcription factor complex BMAL1/CLOCK that activates the Period and Chryptochrome genes (Per1, Per2, Cry1 and Cry2) (Figure 1A). After several hours, PER and CRY proteins form a complex in the cytoplasm, go back to the nucleus and downregulate their own synthesis by inhibiting BMAL1/CLOCK. Once the latter protein complex is inhibited, transcription of PER and CRY stops. Hence, 
BMAL1/CLOCK is no longer inhibited and the cycle starts its process again. The model also includes a positive feedback loop where Bmal1 transcription is positively regulated by PERs and CRYs because the complex PER/CRY also inhibits the transcription of Rev-erb $\alpha$, which inhibits the transcription of Bmal1.

Here, we focus on the coupling between the cell cycle and the circadian clock through the protein WEE1. The combined molecular/population model consists of two coupled systems of equations: one system of ordinary differential equations that describes the molecular dynamics of the cell cycle and the circadian clock, and one system of partial differential equations that describes the growth of a cell population. The molecular model itself is a coupled system of two core networks, one for the circadian clock, and one for the cell cycle.

According to Nagoshi et al. [15], cultured fibroblasts harbor self-sustained and cell autonomous circadian clocks similar to those operative in the neurons of the suprachiasmatic nuclei. Similar results were obtained for yeast, where the regulation of oscillations is not the result of a central oscillator, but rather it emerges from numerous subgraphs with the potential to oscillate with stable periodicity [8]. Also, circadian gene expression continues during cell division and daughter cells resume the rhythms of mother cells after mitosis. We assume that cell cycle divisions do not alter the molecular concentration of the circadian components, neither their rhythms, which can be linked to the fact that daughter cells inherit the same circadian expressions of their mothers.

For the circadian clock, we used the model proposed by Becker-Weimann 


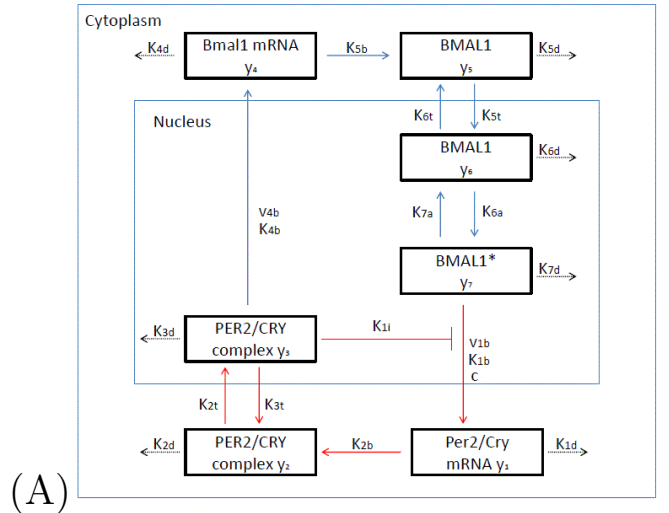

(B)

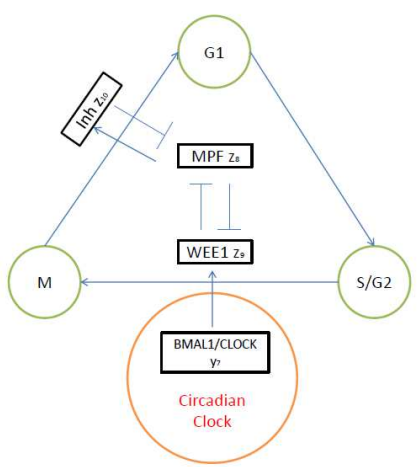

Figure 1: (A) Scheme of the circadian clock network: the activated heterodimer BMAL1/CLOCK (BMAL1 ${ }^{\star}, y_{7}$ ) activates Per2 and Cry genes, which produce Per2/Cry mRNA $\left(y_{1}\right)$. PER2 and CRY proteins are synthesized and bind in the cytosol to form a complex $\left(y_{2}\right)$ to be transported into the nucleus $\left(y_{3}\right)$. This complex inhibits the activity of BMAL1/CLOCK complex, thus destroying its own source of transcription and closing the negative feedback loop. The nuclear complex PER2/CRY $\left(y_{3}\right)$ also activates Bmal1 transcription, which produces an increase in Bmal1 mRNA $\left(y_{4}\right)$, and cytosolic protein concentration $\left(y_{5}\right)$. The BMAL1/CLOCK complex is then transported to the nucleus $\left(y_{6}\right)$, where it is activated. The activated BMAL1/CLOCK complex $\left(\mathrm{BMAL1}^{\star}, y_{7}\right)$ restarts the activation process of Per2/Cry. (B) Schematic representation of the coupling between the cell cycle and the circadian clock through the protein WEE1 $\left(z_{9}\right)[24]$. In the model, the cell cycle is divided into three successive phases G1, S/G2, M. Transitions from one phase to another depend on the activity of $\operatorname{MPF}\left(z_{8}\right)$. For cells to leave G1 and enter S/G2, MPF activity must exceed a fixed threshold $\theta_{1}=0.09$. For cells to leave $\mathrm{S} / \mathrm{G} 2$ and enter M phase, MPF activity must exceed that of WEE1 $\left(z_{9}\right)$. In the M phase, MPF activates its inhibitor $\left(z_{10}\right)$, which represses MPF activity, letting it shut down and forcing the cell to exit mitosis. Division occurs once MPF activity reaches a low threshold level $\theta_{2}=0.06$. Coupling between the cell cycle and the circadian clock is achieved by the transcriptional activation of Wee1, which induces WEE1 activity $\left(z_{9}\right)$ by the active BMAL1/CLOCK complex $\left(y_{7}\right)$. 
and colleagues [32]. It consists of seven nonlinear ordinary differential equations describing the concentrations of Per/Cry mRNA and PER/CRY protein complexes, and Bmal1/Clock mRNA and protein complexes $\left(y_{i}, i=\right.$ $1, \ldots, 7)$.

For the cell cycle, we used a system of three ordinary differential equations based on MPF activity $\left(z_{i}, i=8, \ldots, 10\right)$. This model was inspired by a model by Tyson and Novak [33] (The original model is for fission yeast cell cycle, we adapt the notations for mammalian cell cycle). The core of the Tyson and Novak model is based on the activity of the cyclin-dependent protein kinase complexes CyclinB/Cdk1 (also called MPF for mitosis promoting factor), which are the engine needed to start DNA replication and mitosis. The cell cycle is divided into three phases: G1, S/G2, and M. Transitions from one phase to the other depend on the concentration of MPF and its enemies. When the activity of MPF is high, the cell progresses through the cell cycle; when it is low, the cell blocks its progression. Each phase transition of the cycle is regulated by specific enemies and helpers, which decide whether MPF will win or lose. Transition from G1 to $\mathrm{S}$ is governed by the antagonistic interaction between $\mathrm{MPF}$ and its enemies $\mathrm{APC}^{G_{1}}$ and CKI. In the G2/M transition, the enemy of MPF is the tyrosine kinase WEE1, which can inactivate Cdk1. At cell division, or M to G1 transition, MPF activity shuts down to let the cell exit mitosis and enter the G1 phase. The helper molecule for this transition is the $\mathrm{APC}^{M}$ complex, which promotes the degradation of CyclinB. In the model, three players are included explicitly: $\operatorname{MPF}\left(z_{8}\right)$, WEE1 $\left(z_{9}\right)$ and the inhibitor of MPF $\left(z_{10}\right)$. We supposed that cells enter S/G2 phase when MPF increases above a fixed threshold $\left(\theta_{1}\right)$, enter mitosis 
(M phase) when MPF activity rises above that of WEE1, and divide when MPF reaches back a low threshold level $\left(\theta_{2}\right)$, as it happens during mitosis. Even though the cell cycle model presented here is not quantitative due to the small number of kinetic parameters, it still reproduces a correct qualitative behavior of the cell cycle dynamics. Since we were interested in the effects of coupling the circadian clock to the cell cycle through the protein WEE1, we only considered the antagonistic relation of WEE1 and MPF to avoid simulation artefacts, which may come from other interactions that are not related to our study. Our model reproduces well the evolution of MPF activity, which oscillates in an antagonistic way with the activity of WEE1. Once MPF activity surpasses WEE1 activity, it activates its inhibitor (variable $z_{10}$ in our model, which can be associated to $\mathrm{APC}^{M}$ in the Tyson and Novak model, Figure 1 in [33]) to help shutting down its own activity and forces the cell to exit mitosis. 
The full, 10-variable molecular system, reads

$$
\begin{aligned}
& \frac{\mathrm{d} y_{1}}{\mathrm{~d} t}=\frac{\nu_{1 b}\left(y_{7}+c\right)}{k_{1 b}\left(1+\left(\frac{y_{3}}{k_{1 i}}\right)^{p}\right)+y_{7}+c}-k_{1 d} y_{1}, \\
& \frac{\mathrm{d} y_{2}}{\mathrm{~d} t}=k_{2 b} y_{1}^{q}-k_{2 d} y_{2}-k_{2 t} y_{2}+k_{3 t} y_{3} \\
& \frac{\mathrm{d} y_{3}}{\mathrm{~d} t}=k_{2 t} y_{2}-k_{3 t} y_{3}-k_{3 d} y_{3} \\
& \frac{\mathrm{d} y_{4}}{\mathrm{~d} t}=\frac{\nu_{4 b} y_{3}^{r}}{k_{4 b}^{r}+y_{3}^{r}}-k_{4 d} y_{4} \\
& \frac{\mathrm{d} y_{5}}{\mathrm{~d} t}=k_{5 b} y_{4}-k_{5 d} y_{5}-k_{5 t} y_{5}+k_{6 t} y_{6} \\
& \frac{\mathrm{d} y_{6}}{\mathrm{~d} t}=k_{5 t} y_{5}-k_{6 t} y_{6}-k_{6 d} y_{6}+k_{7 a} y_{7}-k_{6 a} y_{6} \\
& \frac{\mathrm{d} y_{7}}{\mathrm{~d} t}=k_{6 a} y_{6}-k_{7 a} y_{7}-k_{7 d} y_{7} \\
& \frac{\mathrm{d} z_{8}}{\mathrm{~d} t}=\frac{k_{0 m p f} k_{1 m p f}^{n}}{k_{1 m p f}^{n}+z_{8}^{n}+s z_{10}^{n}}\left(1-z_{8}\right)-d_{w e e 1} z_{9} z_{8} \\
& \frac{\mathrm{d} z_{9}}{\mathrm{~d} t}=\frac{k_{a c t w}}{k_{a c t w}+d_{w 1}}\left(c_{w}+C y_{7}\right)+ \\
& \left(\frac{k_{a c t w}}{k_{a c t w}+d_{w 1}}-1\right) \frac{k_{\text {inactw }} z_{8}^{n} z_{9}}{k_{1 w e e 1}^{n}+z_{8}^{n}}-d_{w 2} z_{9}, \\
& \frac{\mathrm{d} z_{10}}{\mathrm{~d} t}=k_{a c t}\left(z_{8}-z_{10}\right) \text {. }
\end{aligned}
$$

The dynamical variables of the circadian clock are: $y_{1}$ Per2 or Cry mRNA and proteins; $y_{2}$ PER2/CRY complex (cytoplasm); $y_{3}$ PER2/CRY complex (nucleus); $y_{4}$ Bmal1 mRNA; $y_{5}$ BMAL1 cytoplasmic protein; $y_{6}$ BMAL1 nuclear protein; $y_{7}$ Active BMAL1; The dynamical variables of the cell cycle are: $z_{8}$ Active MPF; $z_{9}$ Active WEE1; $z_{10}$ Active MPF inhibitor.

For the cell population system, we used age-structured equations described in the next Section. The molecular model entrains the cell population 
(A)

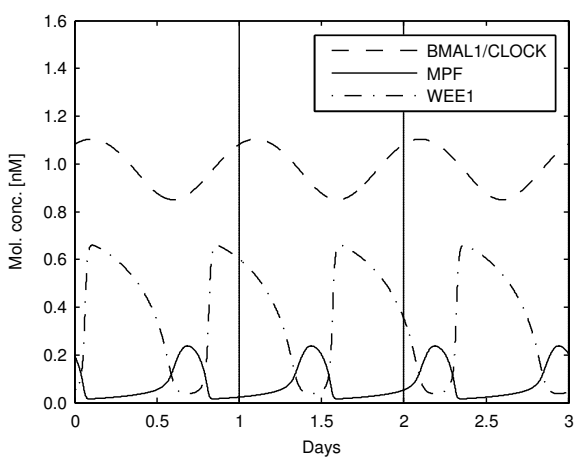

(B)

(C)

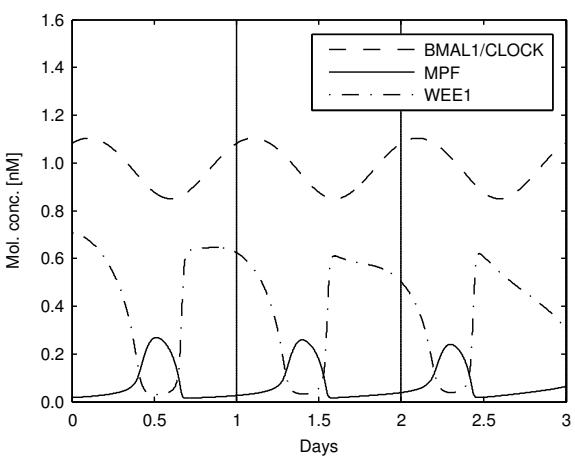

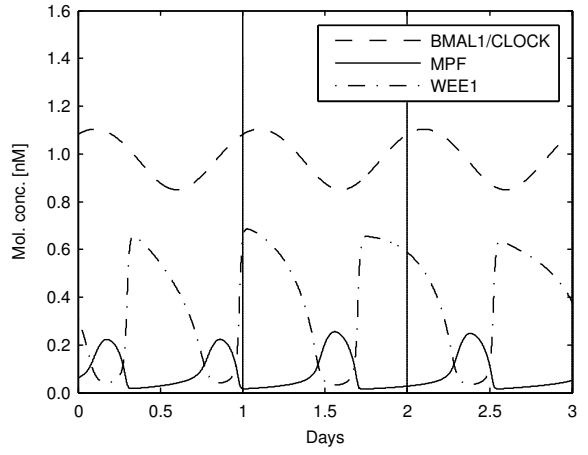

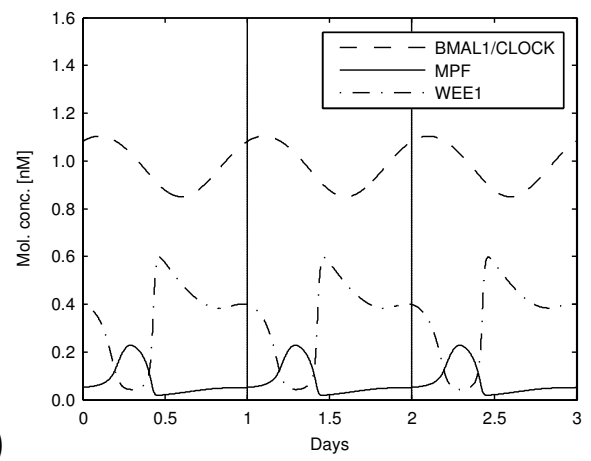

Figure 2: Cell cycle dynamics with coupling to the circadian clock. Autonomous period of the cell cycle is equal to $18 \mathrm{~h}$ in this example. Increasing the coupling strength tends to regulate the cell cycle to $24 \mathrm{~h}$. Coupling strength: (A) 0, (B) 0.5, (C) 1 , (D) 1.5 .

system through cell cycle phase transition rates, which depend on an average molecular state of the cells.

We first studied the influence of the coupling strength between the circadian clock and the cell cycle. The coupling describes the BMAL1/CLOCKmediated rate of WEE1 activation (parameter $C$ in equation 9). Cell cycle durations reported in the literature range from around $8 \mathrm{~h}$ for fast dividing lymphocytes to more than $60 \mathrm{~h}$ for slow tumor cells [34]. Thus, characteristic division times of most mammalian cells coincide with the $24 \mathrm{~h}$ period of the day. To see how cells could entrain to the circadian clock period, we 
chose a cell cycle with an autonomous period (period without coupling to the circadian clock) close, but not equal to $24 \mathrm{~h}$. The cell cycle period was set by scaling the time in the cell cycle equations to obtain the right period. This means that all kinetic events (activation and deactivation) are scaled uniformly. We simulated the influence of the coupling on a cell cycle with an autonomous period of $18 \mathrm{~h}$, for different coupling strengths $C=0,0.5,1,1.5$. We observed that when the coupling strength increases, the period of the cell cycle increases (Figure 2). This behavior was expected for two reasons: (i) WEE1 blocks the cell cycle in G2 phase, and hence slows it down, and (ii) the period of entrainment of the circadian clock is longer than the autonomous period of the cell cycle.

We then asked whether coupling to the circadian clock always slows down the cell cycle, or whether it could speed it up. To answer this question, we looked at the influence of the coupling strength on the cell cycle for autonomous cell cycle period ranging from 8 to $60 \mathrm{~h}$. Our simulations led to different modes of locking between the circadian clock and the cell cycle. For certain combinations of coupling strength and autonomous periods, the cell cycle can entrain to the circadian clock with a rational period ratio, referred to as $n: m$ phase-locking or entrainment (Figure 3A). For a $n: m$ locking, the cell divides $n$ times each $m$ days. These regions of the coupling strength/autonomous periods are called Arnold tongues [35]. Arnold tongues show that the cell cycle can phase-lock to a wide range of orders with $m$ up to 5. 1:1 and 1:2 phase-locks have the widest range of entrainment, but other ratios can be found for large coupling strengths, such as 1:2 and 2:3.

For a fixed coupling strength $(C=1.2)$, the graph of the domains of 

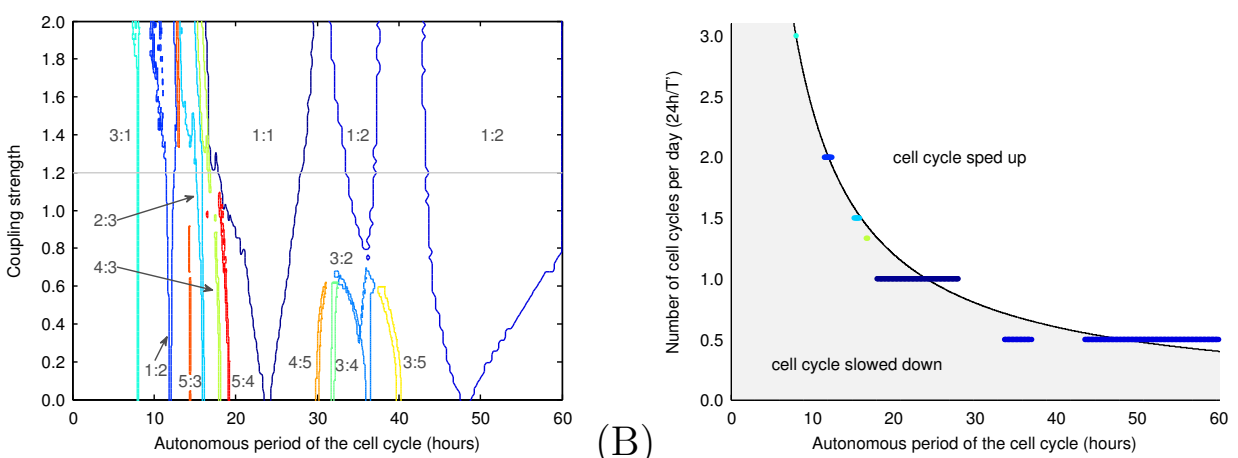

Figure 3: (A) Arnold tongues showing the regions of $n: m$ entrainment for different coupling strengths and autonomous periods. Each region corresponds to an order of entrainment. The order $n: m$ means that cells divide $n$ times every $m$ days. Hence, the 1:1 phase-lock region contains all cell cycles that are entrained to one division per day, or a cycle of 24 $\mathrm{h}$, under an appropriate coupling strength. (B) The $24 h / T$ ' vs $T$ plot for a fixed coupling strength $(C=1.2)$ has a characteristic shape, the devil's staircase [35]. $T$ ' is the period after entrainment by the circadian clock and $T$ is the autonomous period of the cell cycle.

entrainment leads to a devil's staircase (Figure 3B). The devil's staircase shows the frequencies (in number of cell cycles per day) of the phase-locked cell cycles as a function of the autonomous period. Phase-locked frequencies are distributed below (Figure 3B, shaded region) and above the autonomous frequencies (white region), indicating that entrainment by the circadian clock can either slow down, or speed up the cell cycle. The cell cycle is accelerated for intervals of autonomous periods above 24 and $48 \mathrm{~h}$. Therefore, although in our model the circadian clock only acts as a break for cell cycle progression, cells with autonomous periods above 24 or $48 \mathrm{~h}$ can cycle faster under circadian entrainment. 
(A)

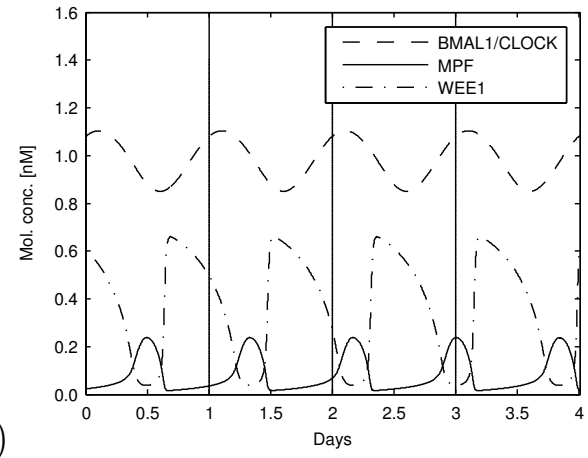

(C)

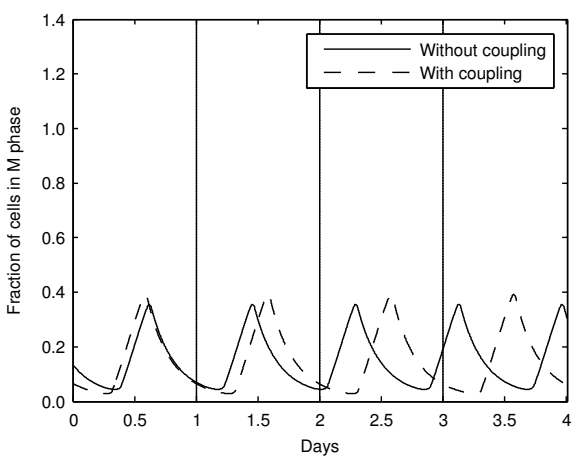

(B)

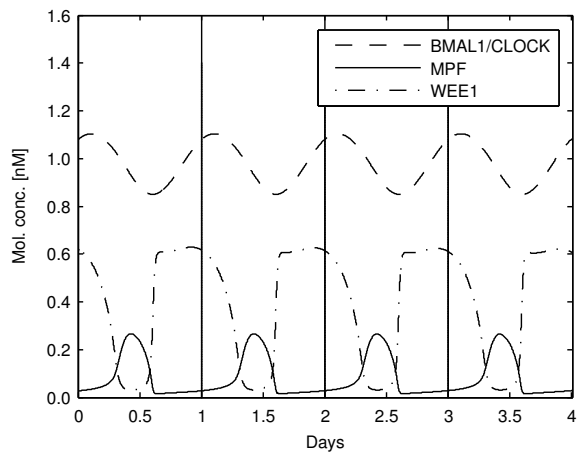

(D)

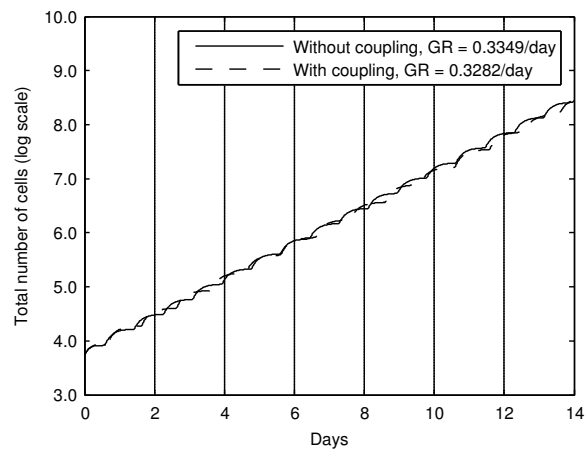

Figure 4: Effects of coupling the circadian clock to the cell cycle with autonomous period of $20 \mathrm{~h}$. (A) Without coupling $(C=0)$ : MPF activity follows a 20-h autonomous cycle. BMAL1/CLOCK period is equal to $24 \mathrm{~h}$. (B) With coupling $(C=1.2)$ : the cell cycle period is entrained to $24 \mathrm{~h}$. (C) The population in $\mathrm{M}$ phase is entrained to $24 \mathrm{~h}$, hence cells have a division cycle of $24 \mathrm{~h}$ instead of $20 \mathrm{~h}$. (D) With coupling, the growth rate does not decrease even though the cell cycle period becomes longer. 

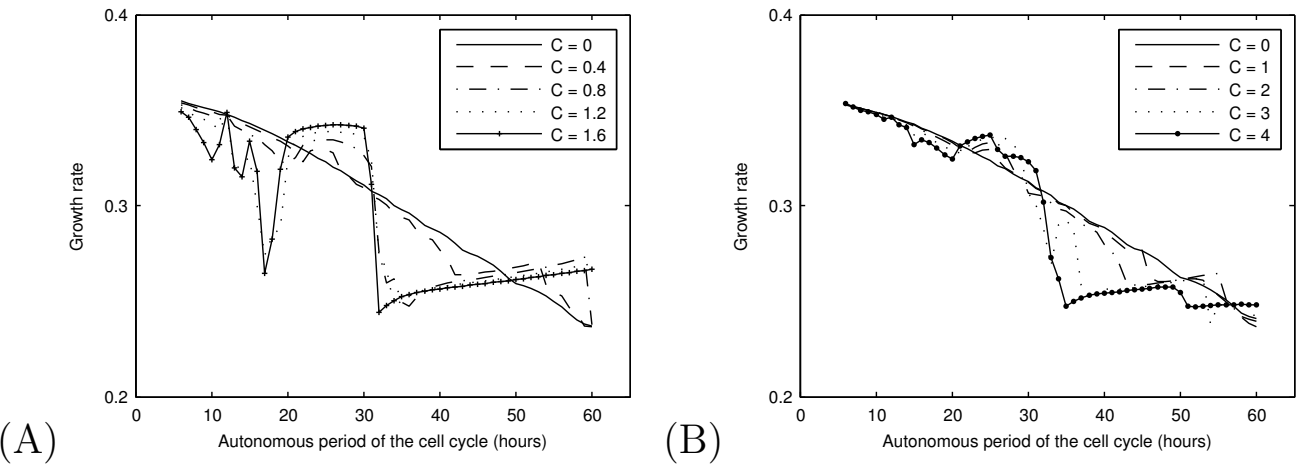

(B)

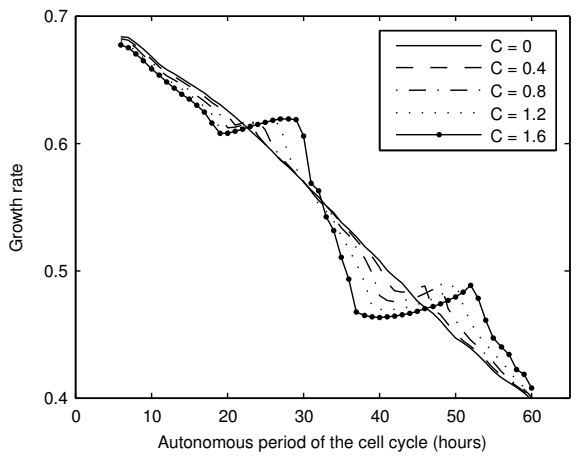

Figure 5: Effects of coupling on the growth rate. (A) Present model. (B-C) Effects of coupling on the growth rate with other models chosen from literature for the circadian clock: Mirsky et al. [36] (B), and Leloup et Goldbeter [37]) (C). 


\section{From molecular concentrations to population growth}

The simulations so far show that the circadian clock could make the cell cycle model run faster or slower, depending on its autonomous period. How does this translate into a net growth rate in a dividing cell population is unclear, and recent analytical results have shown counter-intuitive effects of periodic forcing on growth rates of proliferating cells. There is no systematic inequality when comparing growth rates of a population under circadian control versus a population with a constant, average control [30, 31], but it seems that populations under circadian control that have a cell cycle period close to multiples of $24 \mathrm{~h}$ proliferate faster [38].

We would expect the cell cycle period to be inversely proportional to the growth rate, as in the devil's staircase (Figure 3B). If this were so, the knowledge of the clock-entrained period should be enough to determine the cell population dynamics, without the need of population models. To test that hypothesis, we set up a cell population model entrained by the circadian clock. We used an age-structured model that tracks the time elapsed by cells in each cell cycle phase $[39,40,41]$. We divided the cell cycle model into three phases corresponding to the three phases of the molecular model: G1, $\mathrm{S} / \mathrm{G} 2$, and $\mathrm{M}$ phases. The equations read

$$
\begin{aligned}
& \partial_{t} n_{i}(t, x)+\partial_{x} n_{i}(t, x)+K_{i}(y, z) n_{i}(t, x)=0, \\
& n_{i+1}(t, 0)=\int_{0}^{\infty} K_{i}(y, z) n_{i}(t, x) \mathrm{d} x,
\end{aligned}
$$


for $i=1,2$, and

$$
n_{1}(t, 0)=2 \int_{0}^{\infty} K_{3}(y, z) n_{3}(t, x) \mathrm{d} x
$$

The variable $n_{i}(x, t)$ represents the density of cells in phase $i$. The variable $x$ represents the time spent by a cell in a phase. The parameter $K_{i}$ is the transition rate from phase $i$ to the next phase. The transition between phase $i=3$ and phase $i=1$ marks the cell division, which accounts for the coefficient 2 in the boundary condition for $n_{1}$. Each transition rate $K_{i}$ depends on an average molecular state of the cells. The molecular state is given by the coupled systems of ODEs for the circadian clock and the cell cycle (Equations 1-10). The functional form of the transition rates is a Goldbeter-Koshland function:

$$
K(y, z)=\frac{2 y J_{i}}{z-y+z J_{a}+y J_{i}+\sqrt{\left(z-y+z J_{a}+y J_{i}\right)^{2}-4 y J_{i}(z-y)}} .
$$

This function has been used to generate a switching behavior [33]. If the ratio $y / z$ becomes larger than one, the function switches to the upper state and the transition occurs. $J_{a}$ and $J_{i}$ are two constants that determines the stiffness of the switch, if they tend to zero, the switch tends to a step function. The transition rate from $\mathrm{G} 1(i=1)$ to $\mathrm{S} / \mathrm{G} 2(i=2)$ is switched ON when the concentration of MPF reaches a certain threshold value $\theta_{1}$ that instructs the cell to start DNA synthesis $\left(K_{1}=K\left(z_{8}, \theta_{1}\right)\right)$. The G2 to $\mathrm{M}(i=3)$ transition rate depends on the balance between MPF and WEE1. The cell is blocked in S/G2 and cannot transit to mitosis until MPF concentration exceeds that of WEE1 $\left(K_{2}=K\left(z_{8}, z_{9}\right)\right)$. The transition from M to G1, and cell division, 
occur when the activity of MPF goes back to baseline level $\left(K_{3}=K\left(\theta_{3}, z_{8}\right)\right)$. The total cell number in each phase is given by

$$
N_{i}(t)=\int_{0}^{\infty} n_{i}(t, x) d x
$$

$i=1, \ldots, 3$ and the total cell number is $N(t)=\sum_{i=1}^{3} N_{i}(t)$.

To examine the effect of coupling on the growth rate, we made simulations with and without coupling to the circadian clock. Based on the Arnold tongues for $C=1.2$, the autonomous period of the cell cycle was set to $20 \mathrm{~h}$, inside the 1:1 phase-lock region (Figure 3A). When coupled to the circadian clock, the activity of MPF and WEE1 is well entrained and follows a rhythm of $24 \mathrm{~h}$ (Figure 4A,B). Driven by the new rhythm of MPF and WEE1, the fraction of dividing cells follows a rhythm of $24 \mathrm{~h}$ (Figure 4C). Even though the coupling slows down the cell cycle, the population growth rate stays practically unchanged (Figure $4 \mathrm{D}$ ). This can be justified by the fact that not all cells divide at each cycle. Indeed, there was 0.38 cell division per cell per cycle with coupling, while there was 0.32 cell division per cell per cycle without coupling. Therefore, a longer cell cycle can be compensated by a larger number of division at each cycle, resulting in a higher growth rate than would be inferred from the cell cycle duration only.

To gain more insight on this non-intuitive result, we examined the impact of the coupling strength on the growth rate, for autonomous cell cycle periods ranging from 8 to $60 \mathrm{~h}$. In absence of circadian coupling, the growth rate decreases almost linearly with the cell cycle period (Figure 5A, solid line). In presence of circadian coupling, the growth rate is decreased for most of 
the autonomous cell cycle periods (Figure 5A, non-solid lines). A notable exception is the interval between $20 \mathrm{~h}$ and $31 \mathrm{~h}$, where the growth rate is elevated compared to the growth rate without coupling. This interval corresponds to the range of 1:1 phase-lock (Figure 3). For the larger coupling strengths $(C \geq 1.2)$, the growth rate is almost constant on this interval, as is to be expected from a synchronized population. The elevated growth rate in phase-locked populations is not systematic. For autonomous cell cycle periods above $31 \mathrm{~h}$, which include the 1:2 phase-lock region, the growth rate is almost constant. In this phase-lock region, the cell cycle is entrained on a $48 \mathrm{~h}$ period, and the growth rate is close to the autonomous growth rate at $48 \mathrm{~h}$. These results are in agreement with previous theoretical studies made with population models $[30,31,38]$, which showed that under circadian forcing, the growth rate was elevated near $24 \mathrm{~h}$. Taken together, these results show that the growth rate is related to the entrainment of the molecular cell cycle, but that it is not possible to compare the growth rates with or without coupling.

To test the robustness and genericity of these results, we performed the same simulations on the effect of the coupling strength with two other published models for the circadian clock, one by Mirsky et al. [36], and the other by Leloup et al. [37]. For the Leloup et al. [37] model, we used parameter set 4 . Both models showed the same qualitative result for the impact of the coupling strength on the growth rate. The coupling to the circadian clock increases the growth rate for periods around $24 \mathrm{~h}$, over $48 \mathrm{~h}$ and decreases it elsewhere (Figure 5B,C). 


\begin{tabular}{lll} 
Mutation & \multicolumn{2}{c}{ Circadian clock period } \\
\hline & Experimental & Simulation \\
\hline Per2 & Arrhythmic [36] & Arrhythmic \\
Bmal1 & Arrhythmic [36] & Arrhythmic \\
Cry2 & Rhythmic, long period [36] & Rhythmic, $T=24.2 \mathrm{~h}$ \\
Per2/Cry2 & Rhythmic [42] & Rhythmic, $T=22.7 \mathrm{~h}$ \\
\hline
\end{tabular}

Table 1: Effects of mutations on the period of the circadian clock: comparison between experimental data and simulations.

\section{Circadian clock and cancer}

To investigate the role of the circadian clock in tumor development, we looked at the effect of mutations or deletions of circadian genes on the growth rate. Different types of mutations were examined, namely Per2, Bmal1, Cry2 mutations and Per2/Cry2 double mutations. Per2 and Bmal1 mutations abolished circadian clock rhythmicity, while Cry2 and Per2/Cry2 mutations maintained rhythmicity, in agreement with experimental data (Table A.4 and reference [32], details on simulating mutants are below). Two cases were studied, one considering an autonomous period of the cycle equal to $28 \mathrm{~h}$ and one equal to $20 \mathrm{~h}$. By choosing an appropriate coupling strength $(C=1.2$ for example) to the circadian clock, these two cycles could be entrained to a 1:1 cycle (Figure 3). We looked at changes that occurred after simulating a mutation in the circadian clock. Finally, to have a more global view, we investigated the effect of mutations for autonomous periods ranging from 8 to $60 \mathrm{~h}$.

We studied the effect of mutating Per2 gene by considering that PER2 is a main actor in the negative feedback loop and simulated Per2 mutation by decreasing the rate of PER2/CRY complex formation (we set $k_{2 b}=0.01$ ). 


\section{(A)}
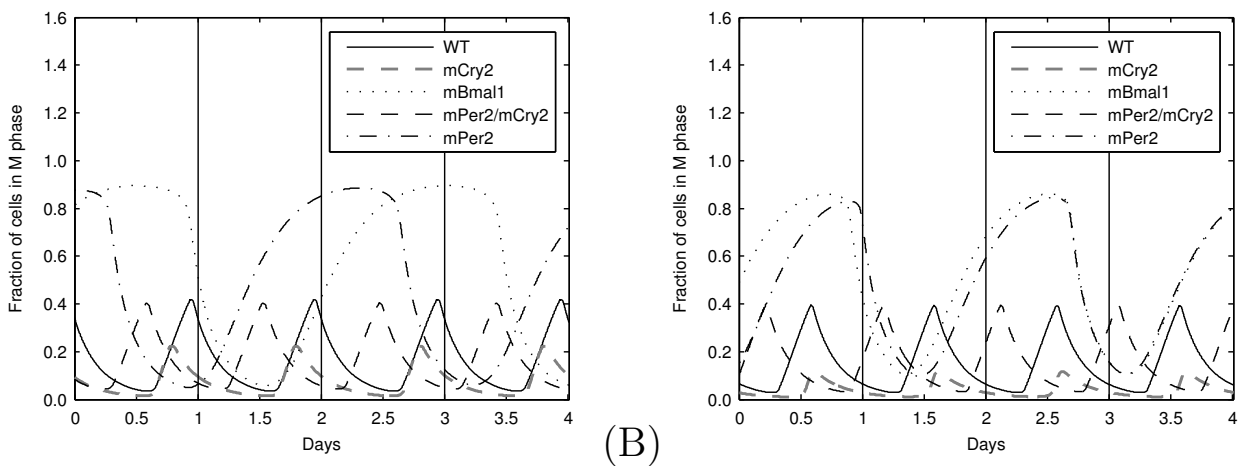

Figure 6: Effects of mutating circadian genes on the fraction of cells entering mitosis. (A) Autonomous period of the cell cycle is equal to $28 \mathrm{~h}$. (B) Autonomous period of the cell cycle is equal to $20 \mathrm{~h}$.

Autonomous period $28 \mathrm{~h}$ Autonomous period $20 \mathrm{~h}$

\begin{tabular}{lrrrr}
\hline mutation & period $(\mathrm{h})$ & g.r. $\left(\mathrm{d}^{-1}\right)$ & period $(\mathrm{h})$ & g.r. $\left(\mathrm{d}^{-1}\right)$ \\
Wild-type & 28.0 & 0.3389 & 20.0 & 0.3282 \\
Per2 & 56.4 & 0.3364 & 38.4 & 0.3954 \\
Bmal1 & 60.0 & 0.2938 & 42.7 & 0.3558 \\
Cry2 & 24.2 & 0.1895 & 24.2 & 0.0986 \\
Per2/Cry2 & 22.6 & 0.3348 & 22.7 & 0.3289 \\
\hline
\end{tabular}

Table 2: Effects of mutations on the period of the M phase (column period) and the growth rate (column g.r.). 
Simulations showed that Per2 mutants have a slower division cycle (Figure $6 \mathrm{~A}, \mathrm{~B}$, dash-dotted lines). This mutation tends to increase the growth rate in the case of $20 \mathrm{~h}$ autonomous cell cycle and keeps it almost equal to that of wild type cells for autonomous period of $28 \mathrm{~h}$ (Table 2). Even though the cell cycle becomes much slower in mutants, in the case of $28 \mathrm{~h}$ autonomous period, 1.05 cell divisions occur during each 56 h-cycle, compared to 0.4 divisions per cycle in wild type cells. This means that for Per2 mutants, some cells must divide more than once during the cycle and explains why the mutants proliferate at the same rate as the wild-type. We also supposed that PER2 activates Bmal1 transcription and simulated Per2 mutation by decreasing Bmal1 transcription rate (we set $\nu_{4 b}=1.5$ ). Similarly, we obtained that Per2 mutants have a slower division cycle and an increased growth rate (Figure 7 dot-dashed lines).

We simulated Bmal1 knockout by setting the transcription rate of Bmal1 $\nu_{4 b}$ equal to 0 . Simulations showed that this mutation tends to slow the cell division cycle for both 20 and $28 \mathrm{~h}$ autonomous period (Figure 6A,B, dotted lines). We observed that this mutation decreases the growth rate for autonomous periods of $28 \mathrm{~h}$ and increases it for autonomous periods of $20 \mathrm{~h}$ (Table 2).

We simulated deficient Cry2 mutants by decreasing the strength of the negative feedback loop (the constant $k_{1 i}$ was increased to $k_{1 i}=0.8$ ). Cry2 mutation preserves the periods of mitotic divisions (Figure 6A,B grey-dashed lines), but decreases the growth rate for both 20 and 28 h-autonomous the cell cycle periods (Table 2).

We simulated Per2/Cry2 double mutants by assuming that Per2 muta- 


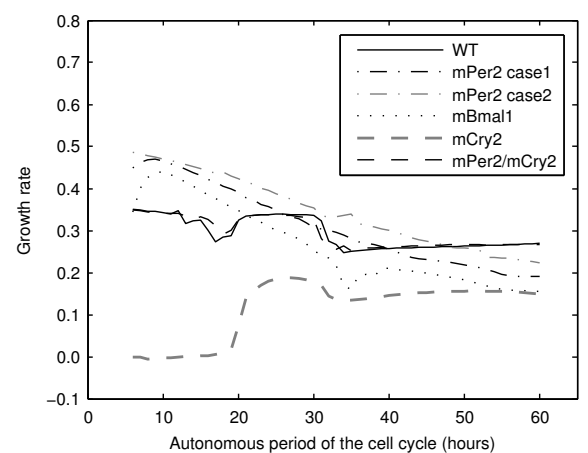

Figure 7: Effects of mutating circadian genes on the growth rate. Mutation of Per2, assuming its role in the negative feedback loop (dot-dashed line), decreases the growth rate for periods ranging from 27 to $31 \mathrm{~h}$, for periods larger than $40 \mathrm{~h}$ and increases it elsewhere. Mutation of Per2 assuming its positive regulation of Bmal1 (grey dot-dashed line) increases the growth rate almost everywhere. Bmal1 knockout (dotted line) increases the growth rate for periods shorter than $21 \mathrm{~h}$ and decreases it elsewhere. Cry2 mutation (grey dashed line) decreases the growth rate everywhere. Per2/Cry2 double mutation (dashed line) maintains a normal proliferation.

tion decreases the rate of PER2/CRY complex formation, and that Cry2 mutation decreases the strength of the negative feedback loop (we set $k_{1 i}=0.8$ and $\left.k_{2 b}=0.01\right)$. Our simulations showed that these double mutants have recovered a mitotic division cycle similar to that of wild type. They also have the same growth rate (Figure 6A,B, dashed lines; Table 2).

Finally, we compared the growth rates for mutants and wild type cells for autonomous cell cycle periods ranging between 8 and 60 h. Perd mutation generally increases the growth rate. Cry2 mutation decreases it, and Bmal1 mutation increases it for autonomous periods less than $21 \mathrm{~h}$ and decreases it elsewhere. Our simulations also predict that Per2/Cry2 double mutants recover a normal proliferation rate and have approximately the same growth rate for all autonomous periods of the cell cycle (Figure 7).

We tested the robustness and the genericity of these results by performing the same simulations on the effect of circadian genes mutations with the 
models proposed by Mirsky et al. [36] and Leloup et al. [37]. Results given by the model proposed by Mirsky et al. [36] were consistent with current model. Namely, Per2 mutation increases the growth rate, Cry1 decreases it, Bmal1 mutation increases it for autonomous periods less than $22 \mathrm{~h}$ and decreases it elsewhere. Simulations on Per and Cry mutations done with the model proposed by Leloup et al. [37] did not show a difference in growth rate compared to the wild type (Figure 8). These results may be explained by the fact that this model is relatively robust to parameter variations. For the parameter set 4 in Leloup et al. [37], the circadian clock was most sensitive to parameters related to Bmal1, for which the effect on the population growth rate was similar to the current model and the model by Mirsky et al. [36].

Taken together, these results predict a differential effect of certain clock gene mutations, depending on the autonomous cell cycle period of the cell population. For instance, Per2 mutant populations grow faster when the autonomous period is shorter than $40 \mathrm{~h}$, but can also grow more slowly if their autonomous period is longer. Other mutations, such as Cry2, systematically slow down the population growth rate.

These results can be explained by looking at the impact of mutations on the MPF/WEE1 dynamics, which dictates the rhythm for cells to enter into mitosis and then divide. Mutation-induced change in BMAL1/CLOCK dynamics, either its period or concentration, directly influences WEE1 activity and the cell cycle dynamics. For example, in case of a $28 \mathrm{~h}$ autonomous period, even though the cell cycle becomes longer for Per2 mutants, the growth rate does not change. Per2 mutation produces an arrhythmic clock with low BMAL1/CLOCK concentration (Figure A.9B, dash-dotted line). A 
comparison of MPF/WEE1 dynamics between mutants and wild type cells shows how the transition rates for the M phase differ (Figure A.10). For Per2 mutants, the transition rate is at a high level for a longer time. This means that even though the cell cycle is longer, much more cells will have the time to enter $\mathrm{M}$ phase and divide. This explains why growth rates are similar in Per2 mutants, even though the cell cycle period is longer. For Cry2 mutants, growth rate decreases. Cry2 mutation leads to higher rates of BMAL1/CLOCK, which in turn increases WEE1 activity (Figure A.9B, grey-dashed line). Increasing WEE1 activity will decrease the activity of MPF (Figure A.9A, grey-dashed line), which means that cells are blocked in G2 phase for a longer time and are prevented from transiting into mitosis. A comparison of the transition rate between wild type and Cry2 mutants shows that transition rate for wild type cells stays on a high level for a longer time than for mutant cells (Figure A.11).

\section{Discussion and Conclusion}

\subsection{Mathematical model for the regulation of the cell cycle by the circadian clock}

We developed a combined molecular/population mathematical model to study how the coupling of the circadian clock to the cell cycle, through the protein WEE1, affects a proliferating cell population. The model has the novelty of combining both, intracellular and population levels. We investigated the influence of coupling on the period of the molecular cell cycle and on the growth rate of the population. The molecular model displays wide ranges of entrainment to the circadian clock, where there is a $n: m$ ratio in 

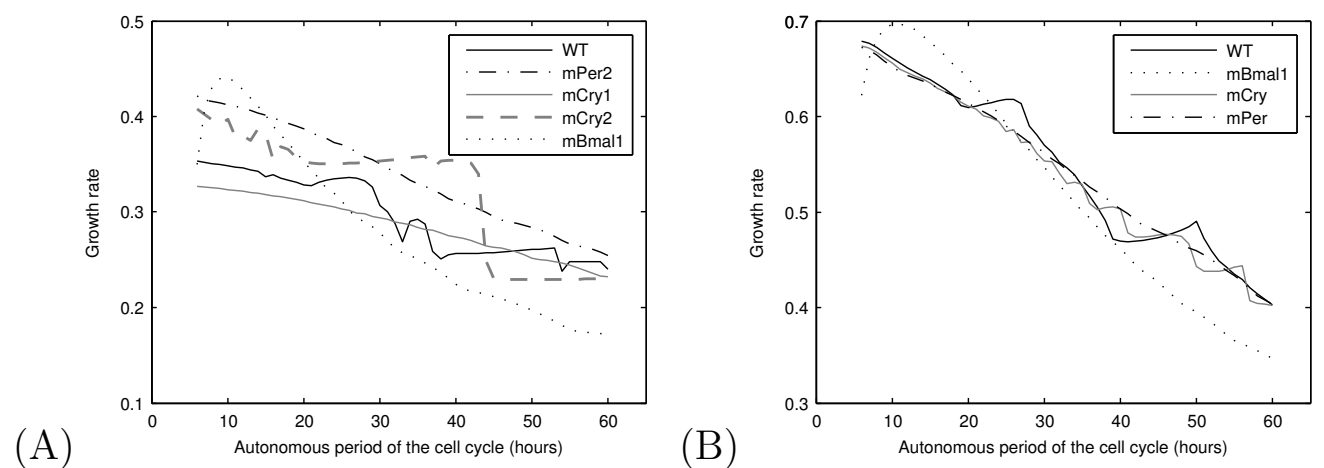

Figure 8: Effects of mutating circadian genes on the growth rate. Simulations were performed with other models chosen from literature for the circadian clock (Mirsky et al. [36] (A), and Leloup and Goldbeter [37] (B)). Both models gave a result for Bmal1 mutants similar to our result, Bmal1 mutation increases the growth rate for autonomous periods less than 22 hours and decreases it elsewhere. For other types of mutations, the first model is more consistent with our model. It gave similar result for Per2 mutants, Per2 mutation increases the growth rate (dot-dashed line (A)). Results for Cry1 mutation were also in agreement with our results. Cry1 mutation (grey solid line (A)) seems to decrease the growth rate for a large interval of autonomous periods. Results for Cry2 mutation are not in agreement with ours, since Cry2 mutation (grey dashed line (A)) seems to increase the growth rate and do not decrease it as it was predicted by our model. Simulations on Per and Cry mutations done with the model proposed by Leloup et al. did not show much difference for the growth rate compared to wild type cells (dot-dashed and grey solid lines (B)). 
the number of cell cycles and the number of circadian oscillations. We found that molecular information about the cell cycle was not always sufficient to predict how the growth rate in a dividing cell population is affected. The combined molecular/population could predict an increase in growth rate in Per2 mutants that could not be explained by the molecular model alone. We used the combined model to look at the influence of circadian clock gene mutations on the population growth rate. We found a differential effect of clock gene mutations, depending on the autonomous cell cycle period of the cell population.

\subsection{Entraining the cell cycle by the circadian clock}

We examined the influence of coupling the cell cycle to the circadian clock on the number of cell cycle divisions per day. We showed that for certain combinations of coupling strength and autonomous periods, the cell cycle can entrain to the circadian clock with a rational period ratio, referred to as $n: m$ phase locking or entrainment. These regions in the coupling strength/autonomous periods space are the Arnold tongues already introduced (Figure 3A). Gérard and colleagues characterized domains of entrainment to 24 and $48 \mathrm{~h}$ periods [28], which correspond to 1:1 and 1:2 phaselocking, respectively. While their model and the current model both predict wide ranges of 1:1 and 1:2 entrainment, there are small differences. In the current model, the 1:2 entrainment region is larger than the 1:1, while the converse is true for the model by Gérard and Goldbeter ([28], their Figure 4B, our Figure 3A). In the current model, 1:2 entrainment is observed at large

coupling strength in a autonomous period range where 3:2 occurs (around autonomous periods of $36 \mathrm{~h}$ ). It looks like that in the Gérard and Goldbeter 
model, the 1:1 entrainment takes over for larger coupling strengths. Recently, Feillet et al. used multispectral imaging of single live cells and mathematical modeling to investigate how the temporal organization of cell division at the single cell level produces daily rhythms at the population level [43]. They demonstrated that there are multiple coexisting robust oscillatory dynamical states of the coupled clock and cell cycle in proliferating mammalian cells, namely 1:1, 5:4 and 3:2 phase locking states. We have characterized a wide range of entrainment modes, including high order phase-locking (3:5, 4:5, $5: 4,5: 3)$, which is consistent with the experimental data of Feillet et al. and which may partly explain the observed quantized cell cycle times discussed previously [27]. The devil's staircase provides a way to predict the frequency of cell divisions as a function of the autonomous cell cycle period. Although the circadian clock acts as a break by activating the inhibitor WEE1, for certain autonomous periods, the cell cycle frequency can still be higher with the clock than without (Figure 3B).

\subsection{Modulation of population growth rate by the clock}

We investigated the influence of coupling on the growth rate for autonomous periods of the cell cycle varying from 8 to $60 \mathrm{~h}$. Clairambault and colleagues [31] showed, using population models, that there is no general inequality between growth rates with and without coupling to the circadian clock. Bernard et al. [38] found that cells under circadian control that have an interdivision time close to multiples of $24 \mathrm{~h}$ proliferate faster. Here, we showed that coupling increases the growth rate for autonomous periods of the cell cycle around $24 \mathrm{~h}$ and above $48 \mathrm{~h}$. For most other periods, the growth rate is decreased. These results could not have been obtained based only on 
the molecular model, which predicted a smaller growth rate for autonomous periods just below $24 \mathrm{~h}$.

\subsection{Effect of mutating clock genes on the growth rate}

We investigated the effect of single or double circadian clock gene mutations on a cell population growth rate. Fu and colleagues showed that loss of Per2 functions increased tumor development [5]. The roles of PER2 in the circadian clock mechanism have been unclear. It is usually considered as a main actor in the negative feedback loop, repressing the activity of BMAL1/CLOCK through the complex PER2/CRY. But some studies also suggest that PER2 activates Bmal1 transcription in an indirect manner $[44,45,46]$. We first examined Per2 mutation by considering that PER2 plays a repressive role in the negative feedback loop. Our simulations are in agreement with experimental results and show that Per2 mutation increases the growth rate for a wide range of autonomous periods of the cell cycle. When a positive action of PER2 on Bmal1 was assumed, we also obtained an increased growth rate for this mutation. We examined Cry2 mutation. We showed that Cry2 mutation decreases the growth rate for almost all periods of the cell cycle. This may explain the experimental results obtained by Matsuo and colleagues, who showed that the weight of regenerating liver in Cry deficient mice was significantly lower than in wild type mice [24].

We examined Per2/Cry2 double mutation. We showed that Per2/Cry2 double mutants recover normal proliferation rates and have similar growth rates for all autonomous periods of the cell cycle. Oster et al. showed that inactivation of Cry2 gene in Per2 mutant mice restored circadian rhythmicity as well as normal clock gene expression patterns [42]. They showed that both 
the period and amplitude of Bmal1 (also of Per1 and Cry1) expressions in Per2/Cry2 double mutant animals were comparable to those of wild types. Hence, if both period and amplitude of Bmal1 are comparable to those of wild type, WEE1 profile will not be changed for these double mutants, preserving normal dynamics for the cell cycle.

We also explored Cry1 mutation and Cry1/Cry2 double mutation, based on the assumption that CRY1 plays a more important role in the negative feedback loop [47]. In the current model, these mutations completely abolished cell proliferation (data not shown). There is no experimental evidence that disruption of the circadian clock can totally prevent cell cycle progression, and it is likely that the cell cycle relies on factors not included in the current model to proceed through division.

\subsection{Robustness of the results}

To test the robustness of our results, we performed our main simulations with two other models for the circadian clock (Mirsky et al. [36], Leloup et al. [37]). We simulated the effect of coupling and circadian genes mutation on the growth rate. Both models show the same qualitative result that we obtain for the impact of the coupling on the growth rate. Results on mutating circadian genes obtained with the model proposed by Mirsky et al. were more consistent with our results. The model proposed by Leloup et al. did not show a difference between mutated and wild type cells. This may be explained by the fact that the model proposed by Mirsky et al. was designed to study the effects of mutating circadian genes, whereas the model proposed by Leloup et al. was designed to generate sustained oscillations, which makes it more robust about parameter variation. 
The current circadian clock model [32] shows limitations in reproducing experimental data that are inherent to models with simplifying assumptions and distinct molecular species lumped together. To check how the simplifying assumptions affect the results, we made a detailed comparison between the current model and the model by Mirsky et al. [36] on the effects of circadian gene mutations. What it is critical in our model is the effect specific mutations on the activity of BMAL1/CLOCK (period and concentration), which regulates directly WEE1 and the cell cycle. Hence, we simulated the effects of circadian gene mutations on BMAL1/CLOCK using Mirsky et al. model (in the same way the authors did it in the original study, Table S3 in [36]) and compared the results with those obtained by the current model. Both models showed similar effects for Bmal1 and Per2 mutations. Bmal1 mutation results in an arrhythmic clock with zero concentration of BMAL1/CLOCK and Per2 mutation results in an arrhythmic clock with a low BMAL1/CLOCK concentration (Figures A.9B,C and Tables A.4, A.5). Consequently, Bmal1 and Per2 mutations have similar effects on the growth rates (Figures 7 and 8A, dotted and dash-dotted lines). Cry2 mutation results in a rhythmic clock with a longer period for both models. However, the period obtained with the model of Mirsky et al. is longer than with the current model ( $\mathrm{T}$ $=32.1 \mathrm{~h}$ vs $\mathrm{T}=24.2 \mathrm{~h}$, Table A.4). The effect on BMAL1/CLOCK concentration is different: the Mirsky et al. model showed a lower concentration compared to wild type, while the current model showed a higher concentration (Figures A.9B,C and Table A.5). This results in different growth rates for the two models (Figures 7 and 8A, grey dashed lines). Per2/Cry2 double mutation results in a rhythmic clock with the current model $(\mathrm{T}=22.7$ 
h), with a concentration similar to wild type, while it results in an arrhythmic clock using the model of Mirsky et al. (Figures A.9B,C and Tables A.4, A.5). Finally, both models gave an arrhythmic clock for Cry1 mutation, with higher BMAL1/CLOCK concentration compared to wild type (Figures A.9B,C, grey solid line).

The main differences between the two models are the effects of Cry2 and Per2/Cry2 mutations. Mirsky et al. predict a longer period for Cry2 mutants, which may be more realistic in the case of lung explants and fibroblasts. The model by Mirsky et al. predicts an arrhythmic clock for Per2/Cry2 double mutation, in contrast to the current model and experiments showing normal rhythmicity for these double mutants [42] . The model by Forger and Peskin also predicts a rhythmic clock for this double mutation [48]. The main limitation of the current model is the way Cry1 mutation and Cry1/Cry2 double mutation are approached. Both are simulated in the same way, by decreasing further the strength of the negative feedback loop. This leads to an arrhythmic clock with high constitutive BMAL1/CLOCK concentration inhibiting cell proliferation, which cannot be supported by experimental data (data not shown). Results given on the growth rate by the model of Mirsky et al. on Cry 1 mutation seems more coherent. Cry 1 mutants have a decreased growth rate compared to wild types for a wide interval of autonomous periods of the cell cycle (Figure 8A, grey solid line).

\subsection{Conclusion}

Combining a molecular model to a population model offers new insight on the influence of the circadian clock on the growth of a cell population. Disruption of the circadian clock can increase or decrease the growth rate, as 
well as the period of mitotic divisions, depending on which clock gene is affected. In some cases, even though the cell cycle slows down, the growth rate can still increase, making the combination of a molecular model and population model unavoidable to study the effect of circadian clock disruption. This can have beneficial impacts on chronotherapy, which aims to develop new strategies in cancer therapies by a better understanding of the circadian clock and its impact on cell proliferation.

The combined model presented in this study is the first step in developing a fully multiscale model for the interaction between the circadian clock and the cell cycle. The multiscale model describes a cell population $p$ structured with a molecular content $(y, z)$ describing the circadian clock and the cell cycle. Heterogeneity among cells can be fully taken into account in a multiscale model, but at the cost of a high-dimensional phase space (here 10D). Even though it has limitations, the current molecular model is simple enough to be amenable to a multiscale description, which, in our view, is essential.

Several studies have shown that the tolerance and the toxicity of drugs varies according to their administration time $[49,50,51]$. Clinical studies showed that compared to standard chemotherapies, chronomodulated chemotherapies, which aim to deliver drugs at an optimal time of the day, could be more efficient and better tolerated by patients $[52,53]$. In a recent work, Bernard et al. [54] used a simple cell population model under chronomodulated treatment and developed a quantitative method to identify biological parameters important for the successful design of a chronotherapy strategy. They found that optimal times depend not only on the circadian status but also on the cell cycle kinetics of the tumor. They suggested that 
the length of the cell cycle is important to determine the best treatment times and intervals. For fast growing tumors, with short S phase, administering a drug that targets the $\mathrm{S}$ phase of the cell cycle at $28.8 \mathrm{~h}$ intervals may be safer than treating at $24 \mathrm{~h}$ intervals, and that for slow growing tumors, with a long $\mathrm{S}$ phase, treating at $24 \mathrm{~h}$ intervals would be the best option. The circadian clock is often disrupted in advanced stage cancers, perhaps because this gives a competitive advantage to growing tumor cells [55]. The current model could be useful to predict how the cell cycle is modified following circadian clock disruption. Combined with the method proposed by bernard et al. [54], this may be of great importance to determine the right time for drug delivery. By taking into account complex interactions between the cell cycle, the circadian clock and the treatment, the combined molecular/population model can be a helpful tool for chronotherapy.

\section{Acknowledgement}

The authors gratefully acknowledge the support of CAMPUS FRANCE and the Lebanese National Council of Scientific Research for funding this work under the PHC Cèdre Grant 30097ZA.

\section{Appendix A. Supplementary figures and tables}




\begin{tabular}{|c|c|c|c|}
\hline Parameters & Values & Units & Description \\
\hline & & & Circadian clock \\
\hline$c$ & 0.01 & $\mathrm{nM}$ & Concentration of constitutive activator \\
\hline$p$ & 8 & Unit less & Hill coefficient \\
\hline$\nu_{1 b}$ & 9 & $\mathrm{nM} h^{-1}$ & Maximal rate of Per2/Cry transcription \\
\hline$k_{1 b}$ & 1 & $\mathrm{nM}$ & Michaelis constant of Per2/Cry transcription \\
\hline$k_{1 d}$ & 0.12 & $h^{-1}$ & Degradation rate of Per $2 /$ Cry mRNA \\
\hline$k_{1 i}$ & 0.56 & $\mathrm{nM}$ & Inhibition constant of Per2/Cry transcription \\
\hline$k_{2 b}$ & 0.3 & $\mathrm{n} M^{-1} h^{-1}$ & Formation rate of cytoplasmic PER2/CRY complex \\
\hline$k_{2 d}$ & 0.05 & $h^{-1}$ & Degradation rate of cytoplasmic PER2/CRY complex \\
\hline$k_{2 t}$ & 0.24 & $h^{-1}$ & Nuclear import rate of PER2/CRY complex \\
\hline$k_{3 t}$ & 0.02 & $h^{-1}$ & Nuclear export rate of PER2/CRY complex \\
\hline$q$ & 2 & Unit less & Number of PER2/CRY2 complex forming subunits \\
\hline$k_{3 d}$ & 0.12 & $h^{-1}$ & Degradation rate of nuclear PER2/CRY complex \\
\hline$\nu_{4 b}$ & 3.6 & $\mathrm{n} M^{-1} h^{-1}$ & Maximal rate of Bmal1 transcription \\
\hline$r$ & 3 & Unit less & Hill coefficient of Bmal1 transcription \\
\hline$k_{4 b}$ & 2.16 & $\mathrm{nM}$ & Michaelis constant of Bmal1 transcription \\
\hline$k_{4 d}$ & 0.75 & $h^{-1}$ & Degradation rate \\
\hline$k_{5 b}$ & 0.24 & $h^{-1}$ & Translation rate of BMAL1 \\
\hline$k_{5 d}$ & 0.06 & $h^{-1}$ & Degradation rate of BMAL1 \\
\hline$k_{5 t}$ & 0.45 & $h^{-1}$ & Nuclear import rate of BMAL1 \\
\hline$k_{6 t}$ & 0.06 & $h^{-1}$ & Nuclear export rate of BMAL1 \\
\hline$k_{6 d}$ & 0.12 & $h^{-1}$ & Degradation rate of nuclear BMAL1 \\
\hline$k_{6 a}$ & 0.09 & $h^{-1}$ & Activation rate of nuclear BMAL1 \\
\hline$k_{7 a}$ & 0.003 & $h^{-1}$ & Deactivation rate of nuclear $\mathrm{BMAL} 1^{\star}$ \\
\hline \multirow[t]{2}{*}{$k_{7 d}$} & 0.09 & $h^{-1}$ & Degradation rate of nuclear BMAL1* \\
\hline & & & Cell cycle \\
\hline$k_{0 m p f}$ & 10 & $h^{-1}$ & Activation rate of $\mathrm{MPF}$ \\
\hline$k_{1 m p f}$ & 0.05 & $\mathrm{nM}$ & Activation rate of MPF \\
\hline$s$ & 20 & $\mathrm{nM}$ & Inhibition constant of MPF \\
\hline$d_{\text {wee } 1}$ & 5 & $h^{-1}$ & Degradation rate \\
\hline$n$ & 2 & Unit less & Hill coefficient \\
\hline$k_{a c t w}$ & 1 & $h^{-1}$ & Activation rate of WEE1 due to BMAL1/CLOCK \\
\hline$d_{w 1}$ & 1 & $n M$ & Michaelis constant \\
\hline$c_{w}$ & 0.5 & $n M$ & Concentration of constant activator \\
\hline$C$ & 0 & $\mathrm{nM}$ & Coupling strength to the circadian clock \\
\hline$k_{\text {inactw }}$ & 200 & $h^{-1}$ & Deactivation rate \\
\hline$k_{1} w e e 1$ & 0.5 & $\mathrm{nM}$ & Michaelis constant \\
\hline$d_{w 2}$ & 1 & $h^{-1}$ & Degradation rate \\
\hline \multirow[t]{2}{*}{$k_{a c t}$} & 0.01 & $h^{-1}$ & Activation rate of MPF inhibitor \\
\hline & & & Population model \\
\hline$\theta_{1}$ & 0.09 & $\mathrm{nM}$ & Threshold value for G1 to S/G2 transition \\
\hline$\theta_{2}$ & 0.06 & $\mathrm{nM}$ & Threshold value for mitotic division ( $M$ to $G 1$ ) \\
\hline$J_{a}$ & 0.1 & Unit less & Stiffness of the switch \\
\hline$J_{i}$ & 0.1 & Unit less & Stiffness of the switch \\
\hline
\end{tabular}

Table A.3: Parameters description.

\begin{tabular}{lll} 
Mutation & \multicolumn{2}{c}{ Circadian clock rhythmicity } \\
\hline & Current & Mirsky et al. \\
\hline Per2 & Arrhythmic & Arrhythmic \\
Bmal1 & Arrhythmic & Arrhythmic \\
Cry2 & Rhythmic,$T=24.2 \mathrm{~h}$ & Rhythmic, $T=32.1 \mathrm{~h}$ \\
Per2/Cry2 & Rhythmic, $T=22.7 \mathrm{~h}$ & Arrythmic \\
Cry1 & Arrhythmic & Arryhthmic \\
\hline
\end{tabular}

Table A.4: Effects of mutations on the period of the circadian clock: comparison between the current model [32] and the model of Mirsky et al. [36]. 
(A)
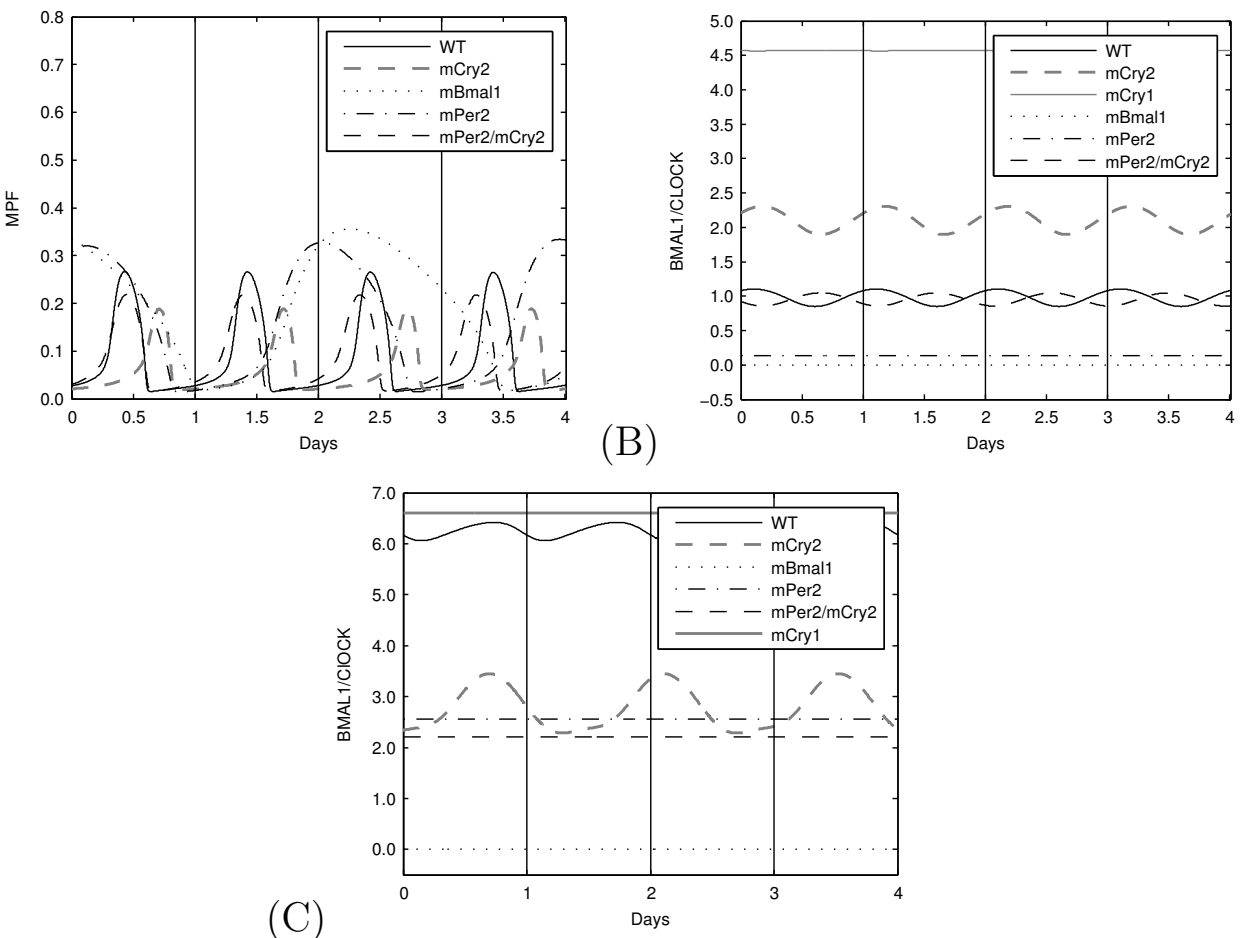

$(\mathrm{C})$

Figure A.9: Effects of mutating circadian genes. (A) MPF activity under different clock mutations. (B) BMAL1/CLOCK activity under different clock mutations, current model [32]. (C) BMAL1/CLOCK activity under different clock mutations, model of Mirsky et al. [36].

\begin{tabular}{lll} 
Mutation & \multicolumn{2}{c}{ BMAL1/CLOCK } \\
\hline & Current & Mirsky et al. \\
\hline Per2 & Lower & Lower \\
Bmal1 & Lower & Lower \\
Cry2 & Higher & Lower \\
Per2/Cry2 & Similar & Lower \\
Cry1 & Higher & Higher \\
\hline
\end{tabular}

Table A.5: Effects of mutations on the BMAL1/CLOCK concentration compared to wild type: comparison between the current model [32] and the model of Mirsky et al. [36]. 
(A)

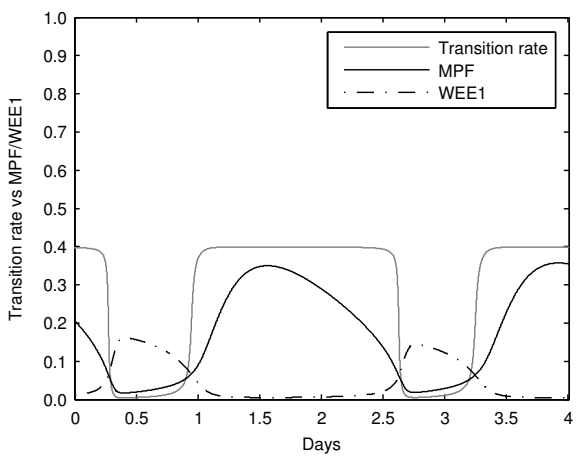

Figure A.10: (A) Per2 mutants. (B) Wild type cells.

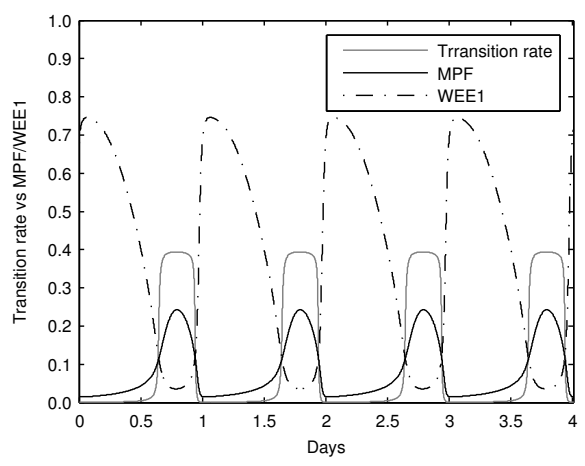

(A)

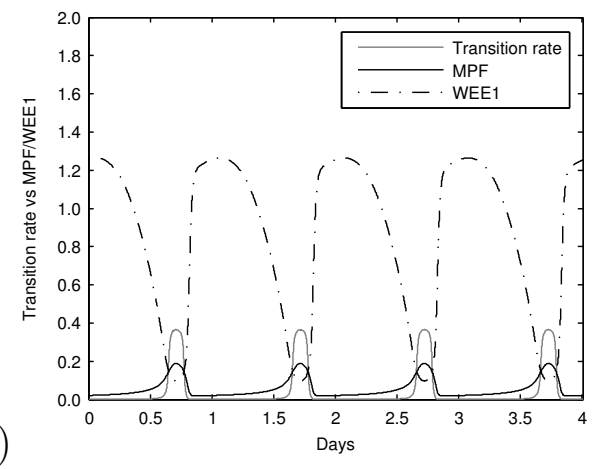

(B)

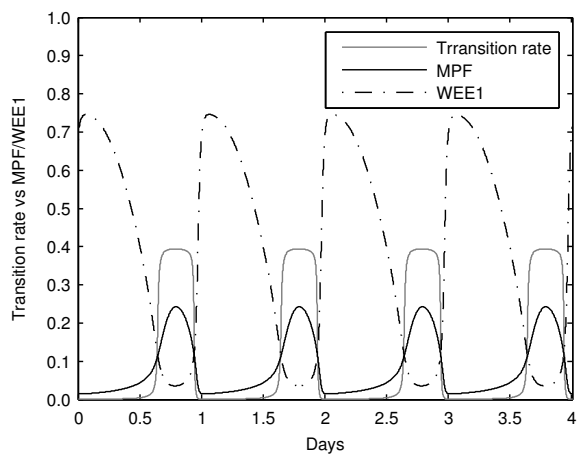

Figure A.11: (A) Cry2 mutants. (B) Wild type cells. 
[1] T. Kubo, K. Ozasa, K. Mikami, K. Wakai, Y. Fujino, Y. Watanabe, T. Miki, M. Nakao, K. Hayashi, K. Suzuki, M. Mori, M. Washio, F. Sakauchi, Y. Ito, T. Yoshimura, A. Tamakoshi, Prospective cohort study of the risk of prostate cancer among rotating-shift workers: findings from the japan collaborative cohort study, Am. J. Epidemiol. 164(6) (2006) 549-555.

[2] E. Filipski, P. Subramanian, J. Carriére, C. Guettier, H. Barbason, F. Lévi, Circadian disruption accelerates liver carcinogenesis in mice, Mutat. Res. 680 (2009) 95-105.

[3] E. Filipski, V. King, X. Li, T. Granda, M. Mormont, X. Liu, B. Claustrat, M. Hastings, F. Lévi, Host circadian clock as a control point in tumor progression, J. Natl. Cancer. Inst. 94 (2002) 690-697.

[4] E. Filipski, F. Delaunay, V. King, M.-W. Wu, B. Claustrat, A. GréchezCassiau, C. Guettier, M. Hastings, F. Lévi, Effects of chronic jet lag on tumor progression in mice, Cancer. Res. 64 (2004) 7879-7885.

[5] L. Fu, H. Pelicano, J. Liu, P. Huang, C. Lee, The circadian gene Period2 plays an important role in tumor suppression and DNA damage response in vivo, Cell 11 (2002) 41-50.

[6] S. Gery, N. Komatsu, L. Baldjyan, A. Yu, D. Koo, H. Koeffler, The circadian gene per1 plays an important role in cell growth and DNA damage control in human cancer cells, Mol. Cell 22 (2006) 375-382.

[7] A. Grèchez-Cassiau, B. Rayet, F. Guillaumond, M. Teboul, F. Delaunay, The circadian clock component BMAL1 is a critical regulator of 
p21 WAF1/CIP1 expression and hepatocyte proliferation, J. Biol. Chem. 283 (2008) 4535-4542.

[8] D. Murray, M. Beckmann, H. Kitano, Regulation of yeast oscillatory dynamics, PNAS 104 (2007) 2241-2246.

[9] B. Tu, A. Kudlicki, M. Rowicka, S. Mcknight, Logic of the yeast metabolic cycle: Temporal compartmentalization of cellular processes, Science 310 (2005) 1152-1158.

[10] J. Edmunds LN, Cellular and molecular bases of biological clocks: models and mechanisms for circadian timekeeping, New York, USA: Springer, 1988.

[11] C. Johnson, Circadian clocks and cell division. What's the pacemaker?, Cell cycle 9 (2010) 3864-3873.

[12] C. Yang, F. Bernardo, G. Dong, S. Golden, A. van Oudenaarden, Circadian gating of the cell cycle revealed in single cyanobacterial cells, Science 327 (2010) 1522-1526.

[13] B. Pando, A. van Oudenaarden, Coupling cellular oscillators-circadian and cell division cycles in cyanobacteria, Curr. Opin. Genet. Dev. 20 (2010) 613-618.

[14] T. Hunt, P. Sassone-Corsi, Riding tandem: Circadian clocks and the cell cycle, Cell 129 (2007) 461-464.

[15] E. Nagoshi, C. Saini, C. Bauer, T. Laroche, F. Naef, et al, Circadian gene 
expression in individual fibroblasts: Cell-autonomus and self-sustained oscillators pass time to daughter cells, Cell 119 (2004) 693-705.

[16] C. Lee, J. Etchegaray, F. Cagampang, A. Loudon, S. Reppert, Posttranslational mechanisms regulate the mammalian circadian clock, Cell. 107 (2001) 855-867.

[17] S. Reppert, D. Weaver, Coordination of circadian timing in mammals, Nature 418 (2002) 935-941.

[18] J. E Eide, M. F Woolf, H. Kang, W. Hurst, F. Camacho, et al, Control of mammalian circadian rhythm by $\mathrm{CKI}_{\varepsilon}$-regulated proteasome-mediated PER2 degradation, Mol. Cell. Biol. 25 (2005) 2795-2807.

[19] L. Cardone, J. Hirayama, F. Giordanao, T. Tamaru, J. Palvino, P. Sassone-Corsi, Circadian clock control by SUMOylation of BMAL1, Science 309 (2005) 1390-1394.

[20] T. Sato, R. Yamada, H. Ukai, J. Baggs, L. Miraglia, T. Kobayashi, et al, Feedback repression is required for mammalian circadian clock function, Nat. Genet. 38 (2006) 212-219.

[21] C. Partch, K. Shields, C. Thompson, C. Selby, A. Sancar, Posttranslational regulation of mammalian circadian clock by chryptochrome and protein phosphatase 5, Proc. Acad. Sci. USA 103 (2006) 10467-10472.

[22] D. Gonze, Modeling circadian clocks: From equations to oscillations, Cent. Eur. J. Biol. 6 (2011) 699-711.

[23] D. Morgan, Principles of cdk regulation, Nature 374 (1995) 131-134. 
[24] T. Matsuo, S. Yamaguchi, S. Mitsui, A. Emi, F. Shimoda, H. Okamura, Control mechanism of the circadian clock for timing of cell division in vivo, Science 302 (2003) 255-259.

[25] Z. Chen, S. McKnight, A conserved DNA damage response pathway responsible for coupling the cell division cycle to the circadian and metabolic cycles, Cell cycle 6(23) (2007) 2906-2912.

[26] A. Chauhan, S. Lorenzen, H. Herzel, S. Bernard, Regulation of mammalian cell cycle progression in the regenrating liver, J. Theor. Biol. 283 (2011) 103-112.

[27] J. Zamborszky, A. Csikasz-Nagy, C. Hong, Computational analysis of mammalian cell division gated by a circadian clock: Quantized cell cycles and cell size, J. Biol. Rhythms 22 (2007) 542-553.

[28] C. Gérard, A. Goldbeter, Entrainment of the mammalian cell cycle by the circadian clock: Modeling two coupled cellular rhythms, PLoS Comput. Biol. 8 (5) (2012) e1002516.

[29] A. Altinok, D. Gonze, F. Lévi, A. Goldbeter, An automaton model for the cell cycle, Interface Focus 1 (2011) 36-47.

[30] J. Clairambault, S. Gaubert, T. Lepoutre, Comparison of Perron and Floquet eigenvalues in age structured cell division models, Math. Comput. Model. 4 (2009) 183-209.

[31] J. Clairambault, S. Gaubert, T. Lepoutre, Circadian rhythm and cell population growth, Math. Comput. Model. 53 (2011) 1558-1567. 
[32] S. Becker-Weimann, J. Wolf, H. Herzel, A. Kramer, Modeling feedback loops of the mammalian circadian oscillator, Biophys. J. 87(5) (2004) 3023-3034.

[33] B. Novak, Z. Pataki, A. Ciliberto, J. Tyson, Mathematical model of the cell division cycle of fission yeast, Chaos 11(1) (2001) 277-286.

[34] Milo, et al., Bionumbers, Nucl. Acids Res. 38 (2010) D750-D753, BNID 100685.

[35] A. Pikovsky, M. Rosenblum, J. Kurths, Synchronization, Cambridge University Press, 2001.

[36] H. Mirsky, A. Liu, D. Welsh, S. Kay, F. Doyle, A model of the cell-autonomous mammalian circadian clock, PNAS 106 (2009) 1110711112.

[37] J.-C. Leloup, A. Goldbeter, Modeling the mammalian circadian clock: Sensitivity analysis and multiplicity of oscillatory mechanisms, J. Theor. Biol. 230 (2004) 541-562.

[38] S. Bernard, H. Herzel, Why do cells cycle with a 24 hour period?, Gen. Info. 17(1) (2006) 72-79.

[39] F. Brikci, J. Clairambault, B. Perthame, Analysis of a molecular structured population model with possible polynamial growth for the cell division cycle, Math. Comp. Model. 47 (2008) 699-713.

[40] F. Brikci, J. Clairambault, B. Ribba, B. Perthame, An age-and-cyclin- 
structured cell population model for healthy and tumoral tissues, J. Math. Biol. 57 (2007) 91-110.

[41] M. Doumic, Analysis of a population model structured by the cells molecular content, Math. Model. Nat. Phenom. 2 (2007) 121-152.

[42] H. Oster, A. Yasui, G. van der Horst, U. Albrecht, Disruption of $m C r y 2$ restores circadian rhythmicity in $m P e r 2$ mutant mice, Genes Dev. 16 (2002) 2633-2638.

[43] C. Feillet, et al., Phase locking and multiple oscillating attractors for the coupled mammalian clock and cell cycle, PNAS 111 (27) (2014) 9828-9833.

[44] L. Shearman, S. Sriram, D. Weaver, E. Maywood, I. Chaves, B. Zheng, K. Kume, C. Lee, T. van der Horst, M. Hastings, S. Reppert, Interacting molecular loops in the mammalian circadian clock, Science 288 (2000) 1013-1019.

[45] W. Yu, M. Nomura, M. Ikeda, Interacting feedback loops within the mammalian clock: BMAL1 is negatively autoregulated and upregulated by CRY1, CRY2, and PER2, Biochem. Biophys. Res. Commun. 290(3) (2002) 933-941.

[46] M. Akashi, A. Okamoto, Y. Tsuchiya, T. Todo, E. Nishida, K. Node, A positive role for PERIOD in mammalian circadian gene expression, Cell Rep. doi:http://dx.doi.org/10.1016/j.celrep.2014.03.072. 
[47] S. Langmesser, T. Tallone, A. Bordon, S. Rusconi, U. Albrecht, Interaction of circadian clock proteins PER2 and CRY with BMAL1 and CLOCK, BMC Mol. Biol. 9 (2008) 41-57.

[48] D. B. Forger, C. S. Peskin, A detailed predictive model of the mammalian circadian clock, PNAS 100 (25) (2003) 14806-14811.

[49] C. Focan, Circadian rhythms and cancer chemotherapy, Pharmacol. Ther. 67 (1995) 1-52.

[50] W. Hrushesky, G. Bjarnason, The application of circadian chronobiology to cancer chemotherapy, Cancer (1993) 2666-2686.

[51] F. Lévi, Therapeutic implications of circadian rhythms in cancer patients, Novartis Found. Symp. 227 (2000) 136-42.

[52] C. Focan, F. Lévi, F. Kreutz, Continuous delivery of venous 5fluorouracil and arterial 5-fluorodeoxyuridine for hepatic metastases from colorectal cancer: feasibility and tolerance in a randomized phase II trial comparing flat versus chronomodulated infusion, Anticancer Drugs 10 (1999) 385-392.

[53] C. Focan, F. Kreutz, D. Focan-Henrard, N. Moeneclaey, Chronotherapy with 5-fluorouracil, folinic acid and carboplatin for metastatic colorectal cancer; an interesting therapeutic index in a phase II trial, Eur. J. Cancer 36 (2000) 341-347.

[54] S. Bernard, B. C. Bernad, F. Lévi, H. Herzel, Tumor growth rate determines the timing of optimal chronomodulated treatment schedules, PLoS Comp. Biol. 6 (2010) e1000712. 
[55] C. Savvidis, M. Koutsilieris, Circadian rhythm disruption in cancer biology, Mol. Med. 18(1) (2012) 1249-1260. 